\title{
MULTIPLICATIVE SUBSETS OF ATOMS
}

\author{
Ashley Rand \\ Received: 6 May 2015; Revised: 24 June 2015 \\ Communicated by Abdullah Harmancı
}

\begin{abstract}
A reduced, cancellative, torsion-free, commutative monoid $M$ can be embedded in an integral domain $R$, where the atoms (irreducible elements) of $M$ correspond to a subset of the atoms of $R$. This fact was used by J. Coykendall and B. Mammenga to show that for any reduced, cancellative, torsion-free, commutative, atomic monoid $M$, there exists an integral domain $R$ with atomic factorization structure isomorphic to $M$. More generally, we show that any "nice" subset of atoms of $R$ can be realized as the set of atoms of an integral domain $T$ that contains $R$. We will also give several applications of this result.
\end{abstract}

Mathematics Subject Classification (2010): 20M14, 20M15, 13G05

Keywords: Cancellative commutative monoid, reduced monoid, atomic monoid, integral domain

\section{Introduction}

Factorization properties in integral domains, from the atomic property to unique factorization, have been studied extensively. One such property is the ascending chain condition on principal ideals (ACCP). It is known that $R$ satisfies ACCP if and only if $R[X]$ satisfies ACCP. Also, there have been several examples from Grams [7] and Zaks [12] showing that the atomic property, where every nonzero nonunit of $R$ can be factored as a finite product of atoms (irreducible elements), is weaker than ACCP. In [2], Anderson, Anderson and Zafrullah posed the natural question, "Is $R[X]$ atomic when $R$ is atomic?". Roitman answered this question in the negative in [10]. He started with $R=F\left[Z,\left\{\frac{Y_{1}}{Z^{n}}, \frac{Y_{2}}{Z^{n}} \mid n \geq 0\right\}\right]$ for a field $F$, and constructed an atomic domain $A$ by adjoining an indeterminate $X_{i}$ and the element $r / X_{i}$ to $R$ for each reducible element $r \in R$. Then each nonzero nonunit of $R$ is either an atom in $A$ or factors as $r=X_{i}\left(r / X_{i}\right)$, a product of two atoms in $A$. Roitman showed that $A[X]$ is not atomic. Using the same construction technique,

This work is a part of the research done for the author's doctoral dissertation [9] written under the direction of D.F. Anderson at the University of Tennessee. 
Roitman later showed that the power series ring $A[[X]]$ also need not be atomic when $A$ is an atomic domain [11].

This paper begins by revisiting results concerning Roitman's construction that are found in [1], [4], and [5]. Anderson and Anderson explored the factorization properties of an element $r$ in $R, R[X, r / X]$, and $R[X]$ in [1]. They observed that if $r$ is an atom in $R$, then $r=X(r / X)$ is not an atom in $R[X, r / X]$. This property is used by Coykendall and Zafrullah in [5] to eliminate finite factorizations into atoms. They show that it is possible to have unique factorization for elements that factor into a finite product of atoms while also having atoms that are not prime. Lastly in [4], Coykendall and Mammenga focused on the factorization structure of the set of elements of $R$ that can be factored into atoms. Seeing that this set is a monoid under multiplication, they also eliminated atoms and constructed an integral domain with atomic structure isomorphic to a given reduced, cancellative, torsion-free, atomic monoid. We will conclude by considering the relationship that emerges among the constructed integral domains.

Throughout, $R$ will denote an integral domain, $\mathcal{A}_{R}$ the set of atoms of $R, \mathcal{A}(R)$ the multiplicative set generated by the atoms and units of $R$, and $\mathbb{C}, \mathbb{R}, \mathbb{Z}, \mathbb{N}, \mathbb{Z}_{n}$, the complex numbers, real numbers, integers, positive integers, and integers modulo $n$, respectively.

\section{Generalization}

We start by generalizing the results in [4]. To this end, we give a few definitions concerning monoids.

Definition 2.1. Let $M$ be a commutative monoid with binary operation + and identity element 0 , and let $a, b, c \in M$.

(1) The monoid $M$ is reduced if whenever $a+b=0$, then $a=0=b$.

(2) The monoid $M$ is cancellative if whenever $a+b=a+c$, then $b=c$.

(3) The monoid $M$ is torsion-free if whenever $n a=n b$ for a positive integer $n$, then $a=b$.

Note that if $M$ is a commutative, cancellative, torsion-free monoid, then $\langle M\rangle:=$ $\{a-b \mid a, b \in M\}$, the group generated by $M$, is a torsion-free abelian group, i.e, $\langle M\rangle$ is an abelian group such that $n a=0$ implies $a=0$ for all $a \in\langle M\rangle$ and $n \in \mathbb{Z} \backslash\{0\}$. In this case, it is also common to say that $M$ is torsionless. It is well-known that a torsionless monoid may be totally-ordered, where the ordering is compatible with the operation ([8, Theorem 22]).

Using these monoid properties, we give a well-known result on graded domains. Recall that a graded domain is an integral domain $R=\oplus_{\alpha \in \Gamma} R_{\alpha}$, where $\Gamma$ is a 
torsionless grading monoid (i.e., $\Gamma$ is commutative, cancellative, and torsion-free), each $R_{\alpha}$ is an additive subgroup of $R$, and $R_{\alpha} R_{\beta} \subseteq R_{\alpha+\beta}$ for $\alpha, \beta \in \Gamma$. The elements of $R_{\alpha}$ are called homogeneous and are said to have degree $\alpha$.

Lemma 2.2. Let $R=\oplus_{\alpha \in \Gamma} R_{\alpha}$ be a graded domain. Then $a b \in R_{\alpha}$ for $0 \neq a, b \in R$ if and only if $a, b$ are homogeneous and $\operatorname{deg}(a)=\alpha-\operatorname{deg}(b)$.

The proof of this lemma relies on the total ordering of $\Gamma$ that is compatible with the operation. We use this lemma repeatedly for $a b \in R$, where $R$ is a subset of a graded domain of the form $R[X, r / X]$. Then Lemma 2.2 restricts us to the homogeneous case with $\operatorname{deg}(a)=-\operatorname{deg}(b)$.

Lemma 2.3. ([1, Proposition 1]) Let $R$ be an integral domain and $0 \neq r \in R$. Then $U(R[X, r / X])=U(R)$ if and only if $r \notin U(R)$.

Lemma 2.4. ([1, Lemma 7$])$ Let $R$ be an integral domain and $0 \neq r \in R$. Then $s \in R$ is an atom in $R[X, r / X]$ if and only if $s$ is an atom in $R$ and not an associate of $r$.

With these lemmas, we can consider the atoms and units in the ring $R\left[\left\{Y_{i}, r_{i} / Y_{i} \mid\right.\right.$ $\left.r_{i} \in S\right\}$ ], where $S \subseteq R \backslash\{0\}$ has arbitrarily many elements and $\left\{Y_{i}\right\}$ is a family of indeterminates. The proofs given here follow the proof of [4, Lemma 3.2] but are included for completeness. Note that $T=R\left[\left\{Y_{i}, r_{i} / Y_{i} \mid r_{i} \in S\right\}\right]$ is a graded domain, graded by $\Gamma=\oplus_{r_{i} \in S} \mathbb{Z}$ with $\operatorname{deg}\left(r \prod Y_{j}^{m_{j}} \prod\left(r_{i} / Y_{i}\right)^{n_{i}}\right)=\left(m_{j}-n_{j}\right)$ for $0 \neq r \in R$. We apply Lemma 2.2 with $T=R$.

Lemma 2.5. Let $R$ be an integral domain, $\emptyset \neq S=\left\{\alpha_{i}\right\} \subseteq R \backslash\{0\}$, and $\left\{Y_{i}\right\}$ be a family of indeterminates. Then $U\left(R\left[\left\{Y_{i}, \alpha_{i} / Y_{i} \mid \alpha_{i} \in S\right\}\right]\right)=U(R)$ if and only if $S \cap U(R)=\emptyset$.

Proof. We prove the finite case by induction on $|S|$. The result holds when $|S|=1$ by Lemma 2.3. For $|S|=n$, let $S=\left\{\alpha_{1}, \ldots, \alpha_{n}\right\}$. Note that $R\left[\left\{Y_{i}, \alpha_{i} / Y_{i} \mid\right.\right.$ $\left.\left.\alpha_{i} \in S\right\}\right]=R\left[\left\{Y_{i}, \alpha_{i} / Y_{i} \mid \alpha_{i} \in S \backslash\left\{\alpha_{n}\right\}\right\}\right]\left[Y_{n}, \alpha_{n} / Y_{n}\right]$, thus by the induction hypothesis $U(R)=U\left(R\left[\left\{Y_{i}, \alpha_{i} / Y_{i} \mid \alpha_{i} \in S \backslash\left\{\alpha_{n}\right\}\right\}\right]\right)$ if and only if $\left(S \backslash\left\{\alpha_{n}\right\}\right) \cap$ $U(R)=\emptyset$. Also, $U\left(R\left[\left\{Y_{i}, \alpha_{i} / Y_{i} \mid \alpha_{i} \in S\right\}\right]\right)=U\left(R\left[\left\{Y_{i}, \alpha_{i} / Y_{i} \mid \alpha_{i} \in S \backslash\left\{\alpha_{n}\right\}\right\}\right]\right)$ if and only if $\alpha_{n} \notin U\left(R\left[\left\{Y_{i}, \alpha_{i} / Y_{i} \mid \alpha_{i} \in S \backslash\left\{\alpha_{n}\right\}\right\}\right]\right)$ by Lemma 2.3. Hence $U\left(R\left[\left\{Y_{i}, \alpha_{i} / Y_{i} \mid \alpha_{i} \in S\right\}\right]\right)=U\left(R\left[\left\{Y_{i}, \alpha_{i} / Y_{i} \mid \alpha_{i} \in S \backslash\left\{\alpha_{n}\right\}\right\}\right]\right)=U(R)$ if and only if $\left(S \backslash\left\{\alpha_{n}\right\} \cup\left\{\alpha_{n}\right\}\right) \cap U(R)=S \cap U(R)=\emptyset$.

Now, suppose that $S$ is infinite and $S \cap U(R)=\emptyset$. We show that $U\left(R\left[\left\{Y_{i}, \alpha_{i} / Y_{i} \mid\right.\right.\right.$ $\left.\left.\left.\alpha_{i} \in S\right\}\right]\right) \subseteq U(R)$, and it follows that $U\left(R\left[\left\{Y_{i}, \alpha_{i} / Y_{i} \mid \alpha_{i} \in S\right\}\right]\right)=U(R)$. Let $r \in U\left(R\left[\left\{Y_{i}, \alpha_{i} / Y_{i} \mid \alpha_{i} \in S\right\}\right]\right)$. Then $r \in U\left(R\left[\left\{Y_{i}, \alpha_{i} / Y_{i} \mid \alpha_{i} \in T\right\}\right]\right)$ for some finite 
subset $T$ of $S$. By the finite case, we have $U\left(R\left[\left\{Y_{i}, \alpha_{i} / Y_{i} \mid \alpha_{i} \in T\right\}\right]\right)=U(R)$ since $T \cap U(R) \subseteq S \cap U(R)=\emptyset$. Thus $r \in U(R)$. For the reverse implication, assume to the contrary that there exists $\alpha \in S \cap U(R)$. Then there exists $\beta \in R$ be such that $\alpha \beta=1$, with $\beta Y_{\alpha}, \frac{\alpha}{Y_{\alpha}} \in R\left[\left\{Y_{i}, \alpha_{i} / Y_{i} \mid \alpha_{i} \in S\right\}\right] \backslash R$ and $\left(\beta Y_{\alpha}\right)\left(\frac{\alpha}{Y_{\alpha}}\right)=\beta \alpha=1$. Hence $\beta Y_{\alpha}, \frac{\alpha}{Y_{\alpha}} \in U\left(R\left[\left\{Y_{i}, \alpha_{i} / Y_{i} \mid \alpha_{i} \in S\right\}\right]\right) \backslash U(R)$.

The next lemma is a similar generalization for Lemma 2.4.

Lemma 2.6. Let $R$ be an integral domain, $\emptyset \neq S=\left\{\alpha_{i}\right\} \subseteq R \backslash\{0\}$, and $\left\{Y_{i}\right\}$ be a family of indeterminates. Then $r \in R$ is an atom in $R\left[\left\{Y_{i}, \alpha_{i} / Y_{i} \mid \alpha_{i} \in S\right\}\right]$ if and only if $r$ is an atom in $R$ and $r$ is not an associate in $R$ of any $\alpha_{i} \in S$.

Proof. First, we show that $U(T) \cap R=U(R)$ for $T=R\left[\left\{Y_{i}, \alpha_{i} / Y_{i} \mid \alpha_{i} \in S\right\}\right]$. Clearly $U(R) \subseteq U(T) \cap R$. For the reverse inclusion, let $a \in U(T) \cap R$. Then there exists $b \in T$ such that $a b=1 \in R$. Thus $a, b$ are homogeneous and $\operatorname{deg}(b)=$ $-\operatorname{deg}(a)=0$ by Lemma 2.2. It follows that $b \in R$, and hence $a \in U(R)$. Thus $U(T) \cap R \subseteq U(R)$, so $U(T) \cap R=U(R)$.

$(\Rightarrow)$ Let $r \in R$ be an atom in $T$. First we show that $r$ is an atom in $R$. Suppose $r=x y$ for $x, y \in R$. We may assume $x \in U(T)$ since $r$ is an atom in $T$. Then $x \in U(T) \cap R=U(R)$, and thus $r$ is an atom in $R$. Now we show $r$ is not an associate of any $\alpha_{i} \in S$. If $r$ is an associate in $R$ of some $\alpha_{i} \in S$, then there exists $u \in U(R)$ such that $r=u \alpha_{i}=\left(u Y_{i}\right)\left(\alpha_{i} / Y_{i}\right)$ is a nontrivial factorization in $T$. This is a contradiction. Thus $r$ is an atom in $T$ and not an associate of any $\alpha_{i} \in S$.

$(\Leftarrow)$ Conversely, let $r$ be an atom in $R$ that is not an associate in $R$ of any $\alpha_{i} \in S$. Suppose that $r=a b$ for $a, b \in T$. Then $a b=r \in R$; thus $a$ and $b$ are homogeneous and $\operatorname{deg}(a)=-\operatorname{deg}(b)$ by Lemma 2.2. So there exist $0 \neq$ $r_{1}, r_{2} \in R, \alpha_{j_{i}}, \alpha_{m_{s}} \in S$, a family of indeterminates $\left\{Y_{j_{i}}, Y_{m_{s}}\right\}$, and $k_{i}, a_{s} \in \mathbb{N}$ for $1 \leq i \leq n$ and $1 \leq s \leq l$ such that $a=r_{1} Y_{j_{1}}^{k_{1}} \cdots Y_{j_{n}}^{k_{n}}\left(\frac{\alpha_{m_{1}}}{Y_{m_{1}}}\right)^{a_{1}} \cdots\left(\frac{\alpha_{m_{l}}}{Y_{m_{l}}}\right)^{a_{l}}$ and $b=r_{2}\left(\frac{\alpha_{j_{1}}}{Y_{j_{1}}}\right)^{k_{1}} \cdots\left(\frac{\alpha_{j_{n}}}{Y_{j_{n}}}\right)^{k_{n}} Y_{m_{1}}^{a_{1}} \cdots Y_{m_{l}}^{a_{l}}$. If some $j_{i}=m_{s}$, then $Y_{j_{i}}^{k_{i}}\left(\frac{\alpha_{m_{s}}}{Y_{m_{s}}}\right)^{a_{s}}=$ $\alpha_{m_{s}}^{k_{i}}\left(\frac{\alpha_{m_{s}}}{Y_{m_{s}}}\right)^{a_{s}-k_{i}}$ when $a_{s} \geq k_{i}$, and $Y_{j_{i}}^{k_{i}}\left(\frac{\alpha_{m_{s}}}{Y_{m_{s}}}\right)^{a_{s}}=\alpha_{m_{s}}^{a_{s}} Y_{j_{i}}^{k_{i}-a_{s}}$ when $a_{s}<k_{i}$. So we may assume that $j_{i} \neq m_{s}$ for $1 \leq i \leq n$ and $1 \leq s \leq l$. Thus, we have $r=a b=r_{1} Y_{j_{1}}^{k_{1}} \cdots Y_{j_{n}}^{k_{n}}\left(\frac{\alpha_{m_{1}}}{Y_{m_{1}}}\right)^{a_{1}} \cdots\left(\frac{\alpha_{m_{l}}}{Y_{m_{l}}}\right)^{a_{l}} r_{2}\left(\frac{\alpha_{j_{1}}}{Y_{j_{1}}}\right)^{k_{1}} \cdots\left(\frac{\alpha_{j_{n}}}{Y_{j_{n}}}\right)^{k_{n}} Y_{m_{1}}^{a_{1}} \cdots Y_{m_{l}}^{a_{l}}=$ $r_{1} r_{2} \alpha_{j_{1}}^{k_{1}} \cdots \alpha_{j_{n}}^{k_{n}} \alpha_{m_{1}}^{a_{1}} \cdots \alpha_{m_{l}}^{a_{l}}$ with $r_{1}, r_{2}, \alpha_{j_{i}}, \alpha_{m_{s}} \in R$ for $1 \leq i \leq n$ and $1 \leq s \leq l$.

Assume $\alpha_{j_{i}}, \alpha_{m_{s}} \notin U(R)$ for $1 \leq i \leq n$ and $1 \leq s \leq l$. Then, we have $r=\left(r_{1} \alpha_{m_{1}}^{a_{1}} \cdots \alpha_{m_{l}}^{a_{l}}\right)\left(r_{2} \alpha_{j_{1}}^{k_{1}} \cdots \alpha_{j_{n}}^{k_{n}}\right)=\left(r_{1} \alpha_{m_{1}}^{a_{1}} \cdots \alpha_{m_{l}}^{a_{l}} r_{2} \alpha_{j_{1}}^{k_{1}} \cdots \alpha_{j_{n}}^{k_{n}-1}\right) \alpha_{j_{n}}$, with both $r_{1} \alpha_{j_{1}}^{k_{1}} \cdots \alpha_{j_{n}}^{k_{n}} r_{2} \alpha_{m_{1}}^{a_{1}} \cdots \alpha_{m_{l}}^{a_{l}-1}$ and $\alpha_{m_{l}}$ elements of $R$. By assumption $\alpha_{m_{l}} \notin U(R)$ and $r$ is an atom in $R$. It follows that $r_{1} \alpha_{j_{1}}^{k_{1}} \cdots \alpha_{j_{n}}^{k_{n}} r_{2} \alpha_{m_{1}}^{a_{1}} \cdots \alpha_{m_{l}}^{a_{l}-1} \in U(R)$. Thus $k_{1}=k_{2}=\cdots=k_{n}=a_{1}=\cdots=a_{l-1}=a_{l}-1=0$ since the $\alpha_{i}$ are not units. Then $r=\left(r_{1} r_{2}\right) \alpha_{m_{l}}$ with $r_{1} r_{2} \in U(R)$. This is a contradiction to our assumption that $r$ 
is not an associate in $R$ of any $\alpha_{i} \in S$. Thus $a_{l}=0$. It follows that $r=a b=r_{1} r_{2}$ with $a, b \in R$; so we may assume that $a \in U(R) \subseteq U(T)$. Hence $r$ is an atom in $T$.

Now, suppose some of the $\alpha_{i}$ are units. By the above argument, we may assume all of the $\alpha_{i}$ are units with $\alpha_{i} \beta_{i}=1$ for some $\beta_{i} \in R$. It follows that $\frac{1}{Y_{i}}=\beta_{i}\left(\frac{\alpha_{i}}{Y_{i}}\right) \in T$. Recall, $r=\left(r_{1} \alpha_{m_{1}}^{a_{1}} \cdots \alpha_{m_{l}}^{a_{l}}\right)\left(r_{2} \alpha_{j_{1}}^{k_{1}} \cdots \alpha_{j_{n}}^{k_{n}}\right)$ is an atom in $R$ with $r_{1} \alpha_{m_{1}}^{a_{1}} \cdots \alpha_{m_{l}}^{a_{l}}, r_{2} \alpha_{j_{1}}^{k_{1}} \cdots \alpha_{j_{n}}^{k_{n}} \in R$. Thus we may assume $r_{1} \alpha_{m_{1}}^{a_{1}} \cdots \alpha_{m_{l}}^{a_{l}} \in U(R)$. Let $u \in R$ be such that $u\left(r_{1} \alpha_{m_{1}}^{a_{1}} \cdots \alpha_{m_{l}}^{a_{l}}\right)=1$. Then $a\left(u Y_{m_{1}}^{a_{1}} \cdots Y_{m_{l}}^{a_{l}}\left(\frac{1}{Y_{j_{1}}}\right)^{k_{1}} \cdots\left(\frac{1}{Y_{j_{n}}}\right)^{k_{n}}\right)=$ $\left(r_{1} Y_{j_{1}}^{k_{1}} \cdots Y_{j_{n}}^{k_{n}}\left(\frac{\alpha_{m_{1}}}{Y_{m_{1}}}\right)^{a_{1}} \cdots\left(\frac{\alpha_{m_{l}}}{Y_{m_{l}}}\right)^{a_{l}}\right)\left(u Y_{m_{1}}^{a_{1}} \cdots Y_{m_{l}}^{a_{l}}\left(\frac{1}{Y_{j_{1}}}\right)^{k_{1}} \cdots\left(\frac{1}{Y_{j_{n}}}\right)^{k_{n}}\right)$. Now, we have the following $\left(r_{1} Y_{j_{1}}^{k_{1}} \cdots Y_{j_{n}}^{k_{n}}\left(\frac{\alpha_{m_{1}}}{Y_{m_{1}}}\right)^{a_{1}} \cdots\left(\frac{\alpha_{m_{l}}}{Y_{m_{l}}}\right)^{a_{l}}\right)\left(u Y_{m_{1}}^{a_{1}} \cdots Y_{m_{l}}^{a_{l}}\left(\frac{1}{Y_{j_{1}}}\right)^{k_{1}} \cdots\left(\frac{1}{Y_{j_{n}}}\right)^{k_{n}}\right)=$ $u\left(r_{1} \alpha_{m_{1}}^{a_{1}} \cdots \alpha_{m_{l}}^{a_{l}}\right)=1$. Hence $a \in U(T)$ and $r$ is an atom in $T$.

To ensure that the final condition of the previous lemma is satisfied, we require that $S$ be unit closed in $R$. We say that $S \subseteq R$ is unit closed if us $\in S$ for all $s \in S$ and $u \in U(R)$. Notice that if $S$ is unit closed and $r \notin S$, then $r$ is not associates with any $\alpha \in S$. Thus, when $S$ is unit closed, $r \in R$ is an atom in $T=R\left[\left\{Y_{i}, \alpha_{i} / Y_{i} \mid \alpha_{i} \in S\right\}\right]$ if and only if $r$ is an atom in $R$ and $r \notin S$.

We conclude this section with our main result which will be used in several applications in the next section.

Theorem 2.7. Let $R$ be an integral domain and $S$ a subset of atoms of $R$ that is unit closed. Then there exists an integral domain $T$ containing $R$ such that $U(T)=U(R)$ and $\mathcal{A}_{T}=S$.

Proof. Let $T_{0}=R$. Inductively, define

$$
T_{n+1}=T_{n}\left[\left\{Y_{\alpha_{i}^{(n)}}, \alpha_{i}^{(n)} / Y_{\alpha_{i}^{(n)}} \mid \alpha_{i}^{(n)} \in \mathcal{A}_{T_{n}} \backslash S\right\}\right],
$$

where $\left\{Y_{\alpha_{i}^{(n)}}\right\}$ is a family of indeterminates. We know $\mathcal{A}_{T_{n}} \cap U\left(T_{n}\right)=\emptyset$ for all $n \geq 0$, and so $U\left(T_{n}\right)=U\left(T_{n+1}\right)$ by Lemma 2.5. It follows that $U\left(T_{n}\right)=U(R)$ for all $n \geq 0$. Also, $S$ is unit closed so the elements of $S$ are atoms that are not associates with the elements in $\mathcal{A}_{T_{n}} \backslash S$. Thus $S \subseteq \mathcal{A}_{T_{n}}$ for all $n \geq 0$ by Lemma 2.6 .

Let $T=\bigcup T_{n}$. Then $T$ is an integral domain since each $T_{n}$ is an integral domain and $T_{0} \subseteq T_{1} \subseteq T_{2} \subseteq \cdots$. We have shown that $U\left(T_{n}\right)=U(R)$ for all $n \geq 0$; thus $U(T)=U(R)$. It remains to show that $\mathcal{A}_{T}=S$. We have $S \subseteq \mathcal{A}_{T_{n}}$ for all $n \geq 0$, and thus $S \subseteq \bigcap \mathcal{A}_{T_{n}} \subseteq \mathcal{A}_{T}$. For the reverse inclusion, let $r$ be an atom of $T$. Then $r \in T_{n}$ for some $n$. It follows that $r \in \mathcal{A}_{T_{n}}$ since $r \in \mathcal{A}_{T}$ and $U\left(T_{n}\right)=U(T)$. If $r \notin S$, then $r=\left(Y_{r}\right)\left(r / Y_{r}\right)$ is reducible in $T_{n+1} \subseteq T$. This is a contradiction; thus $r \in S$ and $\mathcal{A}_{T}=S$. 


\section{Applications}

We next give several applications of Theorem 2.7. The first example shows that every integral domain is contained in an integral domain (not a field) with no atoms. This type of integral domain has been studied extensively [3] and is called an antimatter domain. In [3, Theorem 2.13], Coykendall, Dobbs and Mullins also prove this result. Our techniques provide a shorter proof with a completely different construction.

Example 3.1. Let $R$ be an integral domain with set of atoms $\mathcal{A}_{R}$. There exists an integral domain $T$ (not a field) containing $R$ such that $T$ has no atoms. If $R$ is not a field, then let $S$ be the empty set and note that $S$ is unit closed. The result follows by Theorem 2.7. If $R$ is a field, note that $R \subseteq R[X]$, and apply the previous argument to $R[X]$.

We also note that the technique of Theorem 2.7 was used in [5] to construct an integral domain where all the finite atom factorizations are unique, but the atoms need not be prime.

Remark 3.2. Coykendall and Zafrullah construct an integral domain with a single, non-prime atom, up to associates [5, Theorem 2.8]. Let $R$ be an integral domain with a non-prime atom $x$. Let $S$ be the set of all associates of $x$, i.e., $S=\{u x \mid$ $u \in U(R)\}$. Note that $S$ is unit closed, and by Theorem 2.7, there exists an integral domain $T$ with $\mathcal{A}_{T}=S$. Thus $x$ is the only atom, up to associates. Note that all elements of $T$ that factor into a finite product of atoms do so uniquely, but $x$ is not prime.

This example leads us to focus on the set of elements in an integral domain that can be written as a finite product of atoms and units. For an integral domain $R$, we will denote this set by $\mathcal{A}(R)$ and say that $\mathcal{A}(R)$ is the multiplicatively closed set generated by the atoms and units of $R$. Notice that for an integral domain $R$, we have $\mathcal{A}(R)=R \backslash\{0\}$ if and only if $R$ is atomic. The set $\mathcal{A}(R)$ has some nice properties. For example, $\mathcal{A}(R)$ is a monoid under multiplication. With this fact in mind, we examine the relationship between monoids and $\mathcal{A}(R)$ by considering semi-group rings. Recall that the semi-group $\operatorname{ring} R[X ; M]=\left\{\sum r_{m} X^{m} \mid r_{m} \in\right.$ $R, m \in M\}$ is an integral domain if and only if $R$ is an integral domain and $M$ is a commutative, cancellative, torsion-free monoid [6, Theorem 8.1]. Moreover, when $R[X ; M]$ is an integral domain, $U(R[X ; M])=\left\{u X^{m} \mid u \in U(R), m\right.$ invertible in $M\}$ [6, Theorem 11.1]. The following lemma shows the relationship between the irreducible elements of $M$ and atoms of $R[X ; M]$. 
Lemma 3.3. ([4, Lemma 3.1]) Let $M$ be a commutative, reduced, cancellative, torsion-free monoid, $R$ be an integral domain, and $m \in M$. Then $m$ is irreducible in $M$ if and only if $X^{m}$ is an atom (irreducible) in the semigroup ring $R[X ; M]$.

With this lemma we are able to strengthen [4, Theorem 3.3]. Instead of an integral domain with the atomic factorization structure isomorphic to a given atomic, commutative, cancellative, reduced, torsion-free monoid $M$, we construct an integral domain $T$ such that $M \cong \mathcal{A}(T)$ by the map $m \mapsto X^{m}$. The only unit of the integral domain $T$ will be 1 . Thus $(\mathcal{A}(T) / \sim)=\mathcal{A}(T) \cong M$, where $\sim$ is the equivalence relation defined by $a \sim b$ if and only if $a$ and $b$ are associates. For the remainder of this paper we use $\sim$ to denote this equivalence relation.

Corollary 3.4. Let $M$ be an atomic, commutative, cancellative, torsion-free, reduced monoid. Then there exists an integral domain $T$ with $\mathcal{A}(T) \cong M$.

Proof. Let $M$ be a monoid that is atomic, commutative, cancellative, torsion-free, and reduced. Then $R=\mathbb{Z}_{2}[X ; M]$ is an integral domain and $U(R)=U\left(\mathbb{Z}_{2}[X ; M]\right)=$ $\left\{r X^{m} \mid r \in U\left(\mathbb{Z}_{2}\right), m\right.$ invertible in $\left.M\right\}=\{1\}[6$, Theorem 11.1]. By Lemma 3.3, we know that $\left\{X^{m} \mid m \in \mathcal{A}_{M}\right\} \subseteq \mathcal{A}_{R}$. Let $S=\left\{X^{m} \mid m \in \mathcal{A}_{M}\right\}$ and note that $S$ is unit closed since $U(R)=\{1\}$. Then there exists an integral domain $T$ such that $\mathcal{A}_{T}=S$ by Theorem 2.7. It follows that $M \cong \mathcal{A}(T)$ by the monoid isomorphism $m \mapsto X^{m}$.

This corollary gives a partial answer to the following question: If $M$ is an atomic, commutative, cancellative monoid, when does there exist an integral domain $R$ such that $\mathcal{A}(R) \cong M$ ? In general, $\mathcal{A}(R)$ is an atomic, commutative, cancellative monoid, but need not be reduced or torsion-free. It is easy to see that $\mathcal{A}(R)$ is not torsionfree if the characteristic of $R$ is not 2 since $(-1)^{2}=1^{2}$, but $-1 \neq 1$, and $\mathcal{A}(R)$ is reduced if and only if $U(R)=\{1\}$. For example, $\mathcal{A}(R)$ is an atomic, commutative, cancellative monoid that is neither torsion-free nor reduced for the integral domain $R=\mathbb{R}+X \mathbb{C}[[X]]$.

The next example shows that for an integral domain $R$ such that $\mathcal{A}(R) / \sim$ is a torsion-free monoid, there exists an integral domain $T$ with $\mathcal{A}(T) \cong \mathcal{A}(R) / \sim$.

Example 3.5. ([9, Example 3.2.5]) Let $R$ be an integral domain with set of atoms $\mathcal{A}_{R}$. Let $M$ be a monoid such that $M \cong \mathcal{A}(R) / \sim$. Note that the equivalence class of an atom in $\mathcal{A}(R)$ is represented by an atom of $M$. Assume $M \cong \mathcal{A}(R) / \sim i$ torsionfree. Then by Corollary 3.4 there exists an integral domain $T$ with $U(T)=\{1\}$ such that $\mathcal{A}(T) \cong M$.

It is clear that $M$ is commutative and atomic; so we show that $M$ is reduced and cancellative. Let $a, b, c \in \mathcal{A}(R)$ such that $\bar{a} \bar{b}=1$. Then there exists $u \in U(R)$ such 
that $u a b=1$. It follows that $u a, b \in U(R)$ and $\overline{u a}=\bar{a}=1=\bar{b}$. Thus $M$ is reduced. To show $M$ is cancellative, suppose $\bar{a} \bar{b}=\bar{a} \bar{c}$. Then there exists $u \in U(R)$ such that $a b=u a c$. Hence $b=u c$ by cancellation; so $\bar{b}=\overline{u c}=\bar{c}$. Thus $M$ is cancellative.

We have considered $\mathcal{A}(R)$ as a monoid, now we consider $\mathcal{A}(R)$ as a multiplicatively closed set generated by the atoms of $R$. One property of multiplicatively closed sets that is of interest is the saturated property. Recall that a multiplicatively closed set $S$ is saturated if whenever $a b \in S$ for $a, b \in R$, then $a, b \in S$. Also, the saturation of a multiplicatively closed set $S$ is the smallest saturated set $S^{*}$ that contains $S$, or equivalently, $S^{*}=\{x \in R \mid x y \in S$ for some $y \in R\}$. We now turn back to Roitman's construction $[10,11]$ to adjoin more atoms and maintain atom factorizations rather than creating factorizations that are not atomic. With this alternate use of the construction, we show that every integral domain $R$ can be embedded in an integral domain $T$ with $\mathcal{A}(R) \subseteq \mathcal{A}(T)$ and $\mathcal{A}(T)$ saturated.

Theorem 3.6. Let $R$ be an integral domain. Then there exists an integral domain $T$ such that $R \subseteq T, U(R)=U(T), \mathcal{A}(R) \subseteq \mathcal{A}(T)$, and $\mathcal{A}(T)$ is saturated.

Proof. Let $T_{0}=R$ and $S_{0}$ be the saturation of $\mathcal{A}\left(T_{0}\right)=\mathcal{A}(R)$ in $R$. Let $A_{0}=$ $S_{0} \backslash \mathcal{A}\left(T_{0}\right)$ be the set of nonunit elements in the saturation of $\mathcal{A}\left(T_{0}\right)$ that cannot be factored as a finite product of atoms. Let $T_{1}=R\left[\left\{Y_{i}^{(0)}, a_{i}^{(0)} / Y_{i}^{(0)} \mid a_{i}^{(0)} \in A_{0}\right\}\right]$, where $\left\{Y_{i}^{(n)}\right\}$ is a family of indeterminates for each $n \geq 0$. Then $A_{0} \subseteq \mathcal{A}\left(T_{1}\right)$ since $a_{i}^{(0)}=Y_{i}^{(0)}\left(a_{i}^{(0)} / Y_{i}^{(0)}\right)$ for all $a_{i}^{(0)} \in A_{0}$, and $Y_{i}^{(0)}, a_{i}^{(0)} / Y_{i}^{(0)} \in \mathcal{A}\left(T_{1}\right)$. It follows from Lemma 2.5 that $U\left(T_{1}\right)=U\left(T_{0}\right)$ since $A_{0} \cap U(R)=\emptyset$, and $\mathcal{A}\left(T_{0}\right) \subseteq \mathcal{A}\left(T_{1}\right)$ by Lemma 2.6. Finally, $S_{0} \subseteq \mathcal{A}\left(T_{1}\right)$ by construction. Inductively define $S_{n}$ to be the saturation of $\mathcal{A}\left(T_{n}\right), A_{n}=S_{n} \backslash \mathcal{A}\left(T_{n}\right)$, and $T_{n+1}=T_{n}\left[\left\{Y_{i}^{(n)}, a_{i}^{(n)} / Y_{i}^{(n)} \mid a_{i}^{(n)} \in A_{n}\right\}\right]$. Then $T_{n}$ is an integral domain for every $n \geq 0$ and $T_{0} \subseteq T_{1} \subseteq T_{2} \subseteq \cdots$; thus $T=\bigcup T_{n}$ is an integral domain. Finally, $\mathcal{A}\left(T_{n}\right) \subseteq S_{n} \subseteq \mathcal{A}\left(T_{n+1}\right)$ and $U\left(T_{n}\right)=U(R)$ for all $n \geq 0$. Hence $\mathcal{A}(T)=\bigcup_{n} S_{n}=\bigcup_{n} \mathcal{A}\left(T_{n}\right)$. We show that $\mathcal{A}(T)=\bigcup_{n} \mathcal{A}\left(T_{n}\right)$ is saturated. Let $a b \in \mathcal{A}(T)$ for $a, b \in T$. Then there exists an $n$ such that $a b \in \mathcal{A}\left(T_{n}\right) \subseteq S_{n}$. Hence $a, b \in S_{n} \subseteq \mathcal{A}\left(T_{n+1}\right) \subseteq \mathcal{A}(T)$ since $S_{n}$ is saturated.

\section{Partial ordering}

In general, for an integral domain $R$ there are many unit closed subsets of $\mathcal{A}_{R}$. Using Theorem 2.7, we can construct a different integral domain for each of these subsets. Before we give the main result, we start with a lemma that indicates the relationship at individual steps of the construction. We use it to show that the ordering of unit closed subsets in $\mathcal{A}(R)$ and the associated constructed integral 
domains reverses, i.e., for unit closed $A \subseteq B \subseteq \mathcal{A}(R)$ with constructed integral domains $T_{A}, T_{B}$, resp., from Theorem 2.7, we have $T_{B} \subseteq T_{A}$.

Lemma 4.1. Let $R$ be an integral domain with set of atoms $\mathcal{A}_{R}$ and $A, B \in\{S \subseteq$ $\mathcal{A}_{R} \mid S$ is unit closed $\}$ with $A \subseteq B$. Then $T_{n}^{B} \subseteq T_{n}^{A}$ for all $n \geq 0$, where $T_{0}^{B}=R$, $T_{n}^{B}=T_{n-1}^{B}\left[\left\{Y_{r}, r / Y_{r} \mid r \in T_{n-1}^{B} \backslash(B \cup U(R))\right\}\right],\left\{Y_{r}\right\}$ is a family of indeterminates, and $T_{n}^{A}$ is defined similarly.

Proof. We proceed by induction on $n$. For $A \subseteq B$, we have $R \backslash(B \cup U(R)) \subseteq R \backslash$ $(A \cup U(R))$. Thus the result holds for $n=1$ and $T_{1}^{B} \subseteq T_{1}^{A}$ by construction. Assume the result holds for $n-1$. Then $T_{n-1}^{B} \subseteq T_{n-1}^{A}$. It follows that $T_{n-1}^{B} \backslash(B \cup U(R)) \subseteq$ $T_{n-1}^{A} \backslash(A \cup U(R))$. Hence $T_{n}^{B}=T_{n-1}^{B}\left[\left\{Y_{r}, r / Y_{r} \mid r \in T_{n-1}^{B} \backslash(B \cup U(R))\right\}\right] \subseteq$ $T_{n-1}^{A}\left[\left\{Y_{r}, r / Y_{r} \mid r \in T_{n-1}^{A} \backslash(A \cup U(R))\right\}\right]=T_{n}^{A}$.

Finally, we state and prove the main result concerning the partial ordering under inclusion of the constructed integral domains.

Theorem 4.2. Let $R$ be an integral domain. Then there exists a function $f:\{S \subseteq$ $\mathcal{A}_{R} \mid S$ is unit closed $\} \rightarrow\left\{T \mid R \subseteq T \subseteq B_{R}=\bigcup B_{n}\right.$ is an integral domain $\}$ such that $f(B) \subseteq f(A)$ whenever $A \subseteq B$.

Proof. We show the desired function is $f:\left\{S \subseteq \mathcal{A}_{R} \mid S\right.$ is unit closed $\} \rightarrow\{T \mid$ $R \subseteq T \subseteq B_{R}=\bigcup B_{n}$ is an integral domain $\}$ defined by $S \mapsto T^{S}$, where $T^{S}$ is the integral domain constructed similarly to the construction in the proof of Theorem 2.7 as follows. Let $T_{0}^{S}=R$ and $T_{n}^{S}=T_{n-1}^{S}\left[\left\{Y_{r}, r / Y_{r} \mid r \in T_{n-1}^{S} \backslash(S \cup U(R))\right\}\right]$, where $\left\{Y_{r}\right\}$ is a family of indeterminates. Then $T^{S}=\bigcup T_{n}^{S}$ is an integral domain since each $T_{n}^{S}$ is an integral domain and $T_{0}^{S} \subseteq T_{1}^{S} \subseteq T_{2}^{S} \subseteq \cdots$. Also, from Theorem 2.7, we have $\mathcal{A}_{T^{S}}=S$. It suffices to show that $f$ satisfies the desired condition, that is, if $A \subseteq B$, for $A, B \in\{S \subseteq \mathcal{A} \mid S$ is unit closed $\}$, then $f(B)=T^{B} \subseteq T^{A}=f(A)$. By Lemma 4.1, $T^{B}=\bigcup T_{n}^{B} \subseteq \bigcup T_{n}^{A}=T^{A}$. Thus $f(B) \subseteq f(A)$.

Note that for an integral domain $R$ all of the constructed integral domains in this section are contained in the integral domain $B$ defined as follows. Let $B_{0}=R$ and $B_{n}=B_{n-1}\left[\left\{Y_{r}, r / Y_{r} \mid r \in B_{n-1}\right\}\right]$, where $\left\{Y_{r}\right\}$ is a family of indeterminates. Then $B_{0} \subseteq B_{1} \subseteq B_{2} \subseteq \cdots$ are integral domains, and thus $B_{R}=\bigcup B_{n}$ is an integral domain.

Acknowledgment. I would like to extend my thanks to David F. Anderson for his guidance, careful proof reading and many helpful comments. Also to the referee for the suggested improvements. 


\title{
References
}

[1] D. D. Anderson and D. F. Anderson, The ring $R[X, r / X]$, in Zero-dimensional Commutative Rings (Knoxville, TN, 1994), 95-113, Lecture Notes in Pure and Appl. Math., 171, Dekker, New York, 1995.

[2] D. D. Anderson, D. F. Anderson and M. Zafrullah, Factorization in integral domains, J. Pure. Appl. Algebra, 69(1) (1990), 1-19.

[3] J. Coykendall, D. E. Dobbs and B. Mullins, On integral domains with no atoms, Comm. Algebra, 27(12) (1990), 5813-5831.

[4] J. Coykendall and B. J. Mammenga, An embedding theorem, J. Algebra, 325 (2011), 177-185.

[5] J. Coykendall and M. Zafrullah, AP-domains and unique factorization, J. Pure Appl. Algebra, 189 (2004), 27-35.

[6] R. Gilmer, Commutative Semigroup Rings, Chicago Lectures in Mathematics, University of Chicago Press, Chicago, IL, 1984.

[7] A. Grams, Atomic rings and the ascending chain condition for principal ideals, Proc. Cambridge Philos. Soc., 75 (1974), 321-329.

[8] D. G. Northcott, Lessons on Rings, Modules and Multiplicities, Cambridge University Press, London, 1968.

[9] A. Rand, Multiplicative Sets of Atoms, PhD dissertation, The University of Tennessee, Knoxville, TN, 2013.

[10] M. Roitman, Polynomial extensions of atomic domains, J. Pure Appl. Algebra, 87(2) (1993), 187-199.

[11] M. Roitman, On the atomic property for power series rings, J. Pure Appl. Algebra, 145(3) (2000), 309-319.

[12] A. Zaks, Atomic rings without a.c.c. on principal ideals, J. Algebra, 74(1) (1982), 223-231.

\author{
Ashley Rand \\ 700 Luther Drive \\ Department of Mathematics \\ Assistant Professor \\ Bethany Lutheran College \\ Mankato, MN \\ e-mail: arand@blc.edu
}

